\title{
COVID-19 and acute myocarditis: current literature review and diagnostic challenges
}

\author{
DTufan Çınar ${ }^{1}$ \\ (D) Mert ilker Hayıroğlu ${ }^{2}$ \\ (D) Vedat Çiçek ${ }^{1}$ \\ (iD) Mehmet Uzun ${ }^{1}$ \\ (D) Ahmet Lütfullah Orhan'
}

1. Department of Cardiology, Haydarpasa Sultan Abdulhamid Han Training and Research Hospital, Istanbul, Turkey. 2. Department of Cardiology, Dr. Siyami Ersek Thoracic and Cardiovascular Surgery Training and Research Hospital, Istanbul, Turkey.

http://dx.doi.org/10.1590/1806-9282.66.S2.48

\section{SUMMARY}

INTRODUCTION: In the current literature, there has been an upsurge of cases of COVID-19-induced acute myocarditis. In this case-based review, we aimed to describe the clinical characteristics, imaging findings, and in-hospital course of acute myocarditis. In addition, the limitations of the myocarditis diagnosis were discussed since only fulminant myocarditis cases have been mentioned in the current literature.

METHODS: We performed a review of the literature of all patients who were diagnosed with COVID-19-induced acute myocarditis using the databases of PubMed, Embase, and the Cochrane.

RESULTS: 16 case reports were found to be related to COVID-19-induced acute myocarditis. We observed that the ECG findings in most of the COVID-19 patients were non-specific, including diffuse ST-segment elevation, non-specific intraventricular conduction delay, sinus tachycardia, and inverted T-waves in anterior leads. Echocardiographic findings of COVID-19-induced acute myocarditis patients ranged from preserved left ventricular ejection fraction (LVEF) without segmental abnormalities to reduced LVEF with global hypokinesia. Interestingly, a few patients with COVID-19-induced acute fulminant myocarditis were steroid-responsive and had an amelioration with glucocorticoid and immunoglobulin therapy.

CONCLUSION: Despite the COVID-19 pandemic worldwide, a limited number of cases has been shared in the current literature. There are a lot of difficulties in the differential diagnosis of acute myocarditis in the context of COVID-19.

KEYWORDS: Coronavirus Infections. Betacoronavirus. Myocarditis. Cardiomyopathies.

\section{INTRODUCTION}

Severe acute respiratory syndrome coronavirus 2 (SARS-CoV-2) is a new variant form of coronavirus that is responsible for the coronavirus disease 2019 (COVID-19). On March 2020, the World Health Organization (WHO) declared COVID-19 a global pandemic after the confirmation of cases in five continents' Although the virus mainly infects the lung epithelial cells causing respiratory signs and symptoms, there has been an upsurge of cases that presented COVID-19induced acute myocarditis. Currently, several mechanisms have been proposed to explain the underlying pathophysiology of COVID-19-related acute myocarditis $^{2}$. It has been suggested that direct viral contact through angiotensin-converting enzyme 2 (ACE-2)

DATE OF SUBMISSION: 23-Jun-2020

DATE OF ACCEPTANCE: 11-Jul-2020

CORRESPONDING AUTHOR: Tufan Çınar

Department of Cardiology, Haydarpasa Sultan Abdulhamid Han Training and Research Hospital, Istanbul, Turkey

Tel: +90 216 542-2010 / Fax: +90 216 542-2020

E-mail:drtufancinar@gmail.com 
signaling pathways might have a role in the myocardial injury ${ }^{3}$. In addition, cytokine release syndrome has been proposed to be the main pathophysiology of COVID-19-induced acute fulminant myocarditis ${ }^{3}$. In this case-based review, we aimed to describe the clinical characteristics, imaging findings, and in-hospital course of acute myocarditis as well as the limitations in regard to myocarditis diagnosis. In addition, we tried to identify the possible underlying mechanisms of COVID-19-related acute myocarditis, whether it is caused by direct viral damage or an inadequate host immune response.

\section{METHODS}

We performed a review of the literature of all patients who were reported to have COVID-19-induced acute myocarditis using the databases of PubMed, Embase, and the Cochrane. All databases were searched in June 2020 using the following keywords: 'COVID-19, acute myocarditis' and 'COVID-19, acute myopericarditis'. In total, 16 case reports were found to be related to COVID-19-induced acute myocarditis. Despite the fact that neither endomyocardial biopsy (EMB) nor cardiac magnetic rezones imaging (CMR) have been performed in several reports, the cases reported under the title of acute myocarditis were included in order to provide the current circumstance in terms of acute myocarditis in the COVID-19 era.

\section{Baseline clinical characteristics}

Tables 1 and 2 summarize the clinical characteristics of COVID-19-induced acute myocarditis patients ${ }^{4-17}$. The majority of the cases were male, and hypertension is the most commonly observed risk factor. In all patients, chest pain and dyspnea were the most common symptoms even though one case presented with atypical symptoms such as neck pain and diarrhea ${ }^{4-8}$. Fever is the most commonly observed sign in COVID-19 patients with acute myocarditis. Patients with lower systolic blood pressure developed cardiogenic shock following admission ${ }^{4}$.

\section{Lab findings}

It is recommended to screen for cardiac injury in patients hospitalized for COVID-19 infection because an early diagnosis has an ultimate role in changing management. Therefore, the measurement of cardiac biomarkers, including troponin and brain natriuretic peptide (BNP), should be performed on admission.
We noted that most cases had elevated troponin and BNP levels on admission. Lymphopenia was present in patients with COVID-19-related acute myocarditis, as expected in a usual viral infection.

\section{Imaging features}

In patients who are suspected of having COVID-19related acute myocarditis, baseline electrocardiography (ECG) should be carried out on admission. In this review, we observed that ECG findings in most of the COVID-19 patients were non-specific, including diffuse ST-segment elevation, non-specific intraventricular conduction delay, sinus tachycardia, and inverted $\mathrm{T}$-waves in anterior leads $\mathrm{s}^{4-8,10,14,17}$. Despite frequent changes in ECG, no specific changes were detected in some cases ${ }^{9,16}$. Because there is the possibility of using a point-of-care ultrasound, it is recommended that echocardiography (ECHO) should be performed if there is a suspicion of COVID-19-related acute myocarditis. In this case-based review, the ECHO findings of patients with COVID-19-induced acute myocarditis ranged from preserved left ventricular ejection fraction (LVEF) without segmental abnormalities to reduced LVEF with global hypokinesia, ${ }^{4,10,14-17}$. Those with a severe and depressed LV function developed cardiogenic shock, thereby having a worse progno$\mathrm{sis}^{4,6}$. Although CMR was not performed in all cases because of prolonged acquisition time and the fact that COVID-19 infection is highly contagious, it showed myocardial edema and sub-epicardial late gadolinium enhancement in some parts of the $\mathrm{LV}^{7-12,16}$. Interestingly, no EMB was performed to confirm the diagnosis in all patients even though it is considered a gold standard. Hemodynamic instability, a contagious risk due to prolonged acquisition time, and coagulopathy were the main reasons for not performing EMB.

\section{In-hospital treatment}

There is no consensus for the prescription of the standard heart failure treatment in addition to the antiviral therapy to patients hospitalized with COVID19-induced acute fulminant myocarditis. Interestingly, intravenous (IV) inotrope treatment and circulatory support have been used in selected cases. Moreover, IV glucocorticoid and immunoglobulin therapy have been implemented in patients with complicated acute myocarditis $^{4,6,10,15,17}$. Most patients received heart failure therapy, antiviral, and antibiotic treatment according to the current literature. A few patients with COVID19-induced acute fulminant myocarditis showed an 
TABLE 1. BASELINE CHARACTERISTICS OF ALL COVID-19 ACUTE MYOCARDITIS PATIENTS

\begin{tabular}{|c|c|c|c|c|c|c|}
\hline $\begin{array}{l}\text { Case, } \\
\text { author }\end{array}$ & Age, sex & Risk factors & Presenting symptoms & $\begin{array}{l}\text { Admission } \\
\text { findings }\end{array}$ & ECG findings & Lab findings \\
\hline $\begin{array}{l}\text { Case 1, } \\
\text { Inciardi } \\
\text { et al. }{ }^{4}\end{array}$ & $53, F$ & - & $\begin{array}{l}\text { Fatigue, } \\
\text { chest pain } \\
\text { and dyspnea }\end{array}$ & $\begin{array}{l}\text { Fever: } 36.6^{\circ} \mathrm{C} \\
\text { HR:100 beats } \\
\text { SBP: } 90 \mathrm{~mm} \mathrm{Hg} \\
\text { DBP:50 } \mathrm{mm} \mathrm{Hg} \\
\text { SpO }_{2}: 98\end{array}$ & $\begin{array}{l}\text { Minimal diffuse } \\
\text { ST-segment elevation } \\
\text { (more prominent in } \\
\text { the inferior and lateral } \\
\text { leads), and an ST-seg- } \\
\text { ment depression with } \\
\text { T-wave inversion in lead } \\
\text { V1 and aVR }\end{array}$ & $\begin{array}{l}\text { WBC: } 8.0 \times 10^{3} / \mu \mathrm{L} \\
\text { Lymphocyte: } 1.4 \times 10^{3} / \mu \mathrm{L} \\
\text { CRP: } 25 \mathrm{mg} / \mathrm{dl} \\
\text { D-dimer: } 500 \mathrm{U} / \mathrm{F} \\
\text { Troponin: } 300 \mathrm{ng} / \mathrm{L} \\
\text { BNP: - }\end{array}$ \\
\hline $\begin{array}{l}\text { Case 2, } \\
\text { Kim et al. }\end{array}$ & $21, \mathrm{~F}$ & - & $\begin{array}{l}\text { Coughing, sputum, } \\
\text { diarrhea, and } \\
\text { shortness of breath }\end{array}$ & - & $\begin{array}{l}\text { Nonspecific intraven- } \\
\text { tricular conduction } \\
\text { delay and multiple } \\
\text { premature ventricular } \\
\text { complexes }\end{array}$ & $\begin{array}{l}\text { WBC: - } \\
\text { Lymphocyte: - } \\
\text { CRP: - } \\
\text { D-dimer: } \\
\text { Troponin: } 1.26 \mathrm{ng} / \mathrm{L} \\
\text { BNP: } 1929 \mathrm{pg} / \mathrm{mL}\end{array}$ \\
\hline $\begin{array}{l}\text { Case 3, } \\
\text { Zeng et al. }{ }^{6}\end{array}$ & $63, M$ & - & $\begin{array}{l}\text { Shortness of breath } \\
\text { and chest tightness } \\
\text { after activity }\end{array}$ & $\begin{array}{l}\text { Fever: } 39.3^{\circ} \mathrm{C} \\
\text { HR: - } \\
\text { SBP: - } \\
\text { DBP: - } \\
\text { Spo }_{2}: 91\end{array}$ & $\begin{array}{l}\text { Sinus tachycardia } \\
\text { and no ST-segment } \\
\text { elevation }\end{array}$ & $\begin{array}{l}\text { WBC: - } \\
\text { Lymphocyte: - } \\
\text { CRP: - } \\
\text { D-dimer: - } \\
\text { Troponin: } 11.37 \mathrm{~g} / \mathrm{L} \\
\text { BNP: } 22.600 \mathrm{pg} / \mathrm{mL}\end{array}$ \\
\hline $\begin{array}{l}\text { Case 4, } \\
\text { Doyen et } \\
\text { al. }^{7}\end{array}$ & $69, M$ & Hypertension & $\begin{array}{l}\text { Cough, } \\
\text { fever, } \\
\text { dyspnea }\end{array}$ & $\begin{array}{l}\text { Fever: } 39.1^{\circ} \mathrm{C} \\
\text { HR: - } \\
\text { SBP: - } \\
\text { DBP: - } \\
\text { Spo }_{2}: 91\end{array}$ & $\begin{array}{l}\text { Inverted T waves in } \\
\text { anterior leads }\end{array}$ & $\begin{array}{l}\text { WBC: } 15.400 \mathrm{~g} / \mathrm{L} \\
\text { Lymphocyte: } 141.9 \times 10^{9} \text { per L } \\
\text { CRP: - } \\
\text { D-dimer: - } \\
\text { Troponin: } 9002 \mathrm{ng} / \mathrm{L} \\
\text { BNP: } 22.600 \mathrm{pg} / \mathrm{mL}\end{array}$ \\
\hline $\begin{array}{l}\text { Case 5, } \\
\text { Trogen et } \\
\text { al. }{ }^{8}\end{array}$ & $17, M$ & $\begin{array}{l}\text { Obesity, } \\
\text { asthma, } \\
\text { spondylolysis }\end{array}$ & $\begin{array}{l}\text { fever and neck pain, } \\
\text { diarrhea }\end{array}$ & $\begin{array}{l}\text { Fever } 103^{\circ} \mathrm{F} \\
\text { HR: } 150 \mathrm{bpm} \\
\text { SBP: } 79 \mathrm{~mm} \mathrm{Hg} \\
\text { DBP: } 66 \mathrm{~mm} \mathrm{Hg} \\
\text { SpO }_{2}: 91\end{array}$ & $\begin{array}{l}\text { Sinus tachycardia and } \\
\text { T-wave inversion par- } \\
\text { ticularly in the inferior } \\
\text { leads }\end{array}$ & $\begin{array}{l}\text { WBC: } 15.4 \mathrm{~g} / \mathrm{dL} \\
\text { Lymphocyte: } 0.9 \times 10^{3} / \mu \mathrm{L} \\
\text { CRP: } 167 \mathrm{mg} / \mathrm{L} \\
\text { D-dimer: } 1218 \mathrm{ng} / \mathrm{mL} \\
\text { Troponin: } 2.97 \mathrm{ng} / \mathrm{ml} \\
\text { BNP: } 2124 \mathrm{pg} / \mathrm{mL}\end{array}$ \\
\hline $\begin{array}{l}\text { Case 6, } \\
\text { Sardari et } \\
\text { al. }{ }^{9}\end{array}$ & $31, M$ & - & $\begin{array}{l}\text { Dyspnea on exertion } \\
\text { and low-grade fever }\end{array}$ & $\begin{array}{l}\text { Fever: } 37.8^{\circ} \mathrm{C} \\
\text { HR: } 70 \mathrm{bpm} \\
\text { SBP: } 110 \mathrm{~mm} \mathrm{Hg} \\
\text { DBP: } 70 \mathrm{~mm} \mathrm{Hg} \\
\text { SpO }_{2}: 98\end{array}$ & Normal findings & $\begin{array}{l}\text { WBC: - } \\
\text { Lymphocyte: - } \\
\text { CRP: } 105 \text { mg/L } \\
\text { D-dimer: - } \\
\text { Troponin: } 2.97 \mathrm{ng} / \mathrm{ml} \\
\text { BNP: - }\end{array}$ \\
\hline $\begin{array}{l}\text { Case } 7, \\
\text { Coyle et } \\
\text { al. }^{10}\end{array}$ & $57, M$ & Hypertension & $\begin{array}{l}\text { Shortness of breath, } \\
\text { fevers, cough, myalgia, } \\
\text { decreased appetite, } \\
\text { nausea and diarrhea }\end{array}$ & $\begin{array}{l}\text { Fever: - } \\
\text { HR: - } \\
\text { SBP: - } \\
\text { DBP: - } \\
\text { Spo }:-^{-}\end{array}$ & $\begin{array}{l}\text { Sinus tachycardia } \\
\text { without ST/T wave } \\
\text { changes }\end{array}$ & $\begin{array}{l}\text { WBC: - } \\
\text { Lymphocyte: Lymphopenia } \\
\text { CRP: Elevated } \\
\text { D-dimer: - } \\
\text { Troponin: Rapid rise } \\
\text { BNP: Rapid rise }\end{array}$ \\
\hline $\begin{array}{l}\text { Case 8, } \\
\text { Beşler and } \\
\text { Arslan }^{11}\end{array}$ & $20, M$ & - & $\begin{array}{l}\text { Febrile sensation } \\
\text { and chest pain }\end{array}$ & $\begin{array}{l}\text { Fever: } 39^{\circ} \mathrm{C} \\
\text { HR: } 111 \mathrm{bpm} \\
\text { SBP: } 149 \mathrm{~mm} \mathrm{Hg} \\
\text { DBP: } 63 \mathrm{~mm} \mathrm{Hg} \\
\text { SpO }_{2}: 97\end{array}$ & - & $\begin{array}{l}\text { WBC: } 6.74 \times 10^{9} \text { per L } \\
\text { Lymphocyte: Lymphopenia } \\
\text { CRP: } 0.0812 \mathrm{~g} / \mathrm{L} \\
\text { D-dimer: - } \\
\text { Troponin: } 0.572 \mathrm{ng} / \mathrm{mL} \\
\text { BNP: } 127 \mathrm{ng} / \mathrm{L}\end{array}$ \\
\hline $\begin{array}{l}\text { Case } 9, \\
\text { Yuan et al.12 }\end{array}$ & $33, M$ & Hypertension & $\begin{array}{l}\text { Chest pain, } \\
\text { fever, and } \\
\text { muscle ache. }\end{array}$ & $\begin{array}{l}\text { Fever: } 37.3^{\circ} \mathrm{C} \\
\text { HR: } 121 \mathrm{bpm} \\
\text { SBP: } 115 \mathrm{~mm} \mathrm{Hg} \\
\text { DBP: } 79 \mathrm{~mm} \mathrm{Hg} \\
\text { Spo: }:-\end{array}$ & Ventricular tachycardia. & $\begin{array}{l}\text { WBC: - } \\
\text { Lymphocyte: - } \\
\text { CRP: - } \\
\text { D-dimer: - } \\
\text { Troponin: - } \\
\text { BNP: - }\end{array}$ \\
\hline $\begin{array}{l}\text { Case 10, } \\
\text { Cizgici } \\
\text { et al. }{ }^{13}\end{array}$ & $78, M$ & - & $\begin{array}{l}\text { Chest pain and } \\
\text { shortness of breath. }\end{array}$ & $\begin{array}{l}\text { Fever: } 37.3^{\circ} \mathrm{C} \\
\text { HR: } 150 \mathrm{bpm} \\
\text { SBP: } 115 \mathrm{~mm} \mathrm{Hg} \\
\text { DBP: } 79 \mathrm{~mm} \mathrm{Hg} \\
\text { SpO }_{2}:-\end{array}$ & $\begin{array}{l}\text { Atrial fibrillation with } \\
150 \text { beats/minute and } \\
\text { concave ST elevation } \\
\text { except for aVR lead }\end{array}$ & $\begin{array}{l}\text { WBC: Leukocytosis } \\
\text { Lymphocyte: Lymphopenia } \\
\text { CRP: } 94.6 \mathrm{mg} / \mathrm{L} \\
\text { D-dimer: - } \\
\text { Troponin: } 998.1 \mathrm{ng} / \mathrm{L} \\
\text { BNP: } 127 \mathrm{ng} / \mathrm{L}\end{array}$ \\
\hline $\begin{array}{l}\text { Case 11, } \\
\text { Asif and } \\
\text { Ali }^{14}\end{array}$ & $64, M$ & $\begin{array}{l}\text { Hypertension, } \\
\text { hyperlipid- } \\
\text { emia }\end{array}$ & Dyspnea & $\begin{array}{l}\text { Fever: - } \\
\text { HR: } 80 \mathrm{bpm} \\
\text { SBP: } 85 \mathrm{~mm} \mathrm{Hg} \\
\text { DBP: } 50 \mathrm{~mm} \mathrm{Hg} \\
\text { Spo }_{2}: 70\end{array}$ & $\begin{array}{l}\text { Non-specific T wave } \\
\text { changes }\end{array}$ & $\begin{array}{l}\text { WBC: Leukocytosis } \\
\text { Lymphocyte: Lymphopenia } \\
\text { CRP: } 94.6 \mathrm{mg} / \mathrm{L} \\
\text { D-dimer: - } \\
\text { Troponin: } \\
\text { O.17 ng/ml. } \\
\text { BNP: - }\end{array}$ \\
\hline
\end{tabular}


TABLE 1.

$($ CONCLUSION)

\begin{tabular}{|c|c|c|c|c|c|c|}
\hline $\begin{array}{l}\text { Case, } \\
\text { author }\end{array}$ & Age, sex & Risk factors & Presenting symptoms & $\begin{array}{l}\text { Admission } \\
\text { findings }\end{array}$ & ECG findings & Lab findings \\
\hline $\begin{array}{l}\text { Case 12, } \\
\text { Asif and } \\
\text { Ali14 }\end{array}$ & $71, \mathrm{~F}$ & $\begin{array}{l}\text { Multiple } \\
\text { myeloma }\end{array}$ & $\begin{array}{l}\text { Fever, cough, and } \\
\text { dyspnea }\end{array}$ & $\begin{array}{l}\text { Fever: - } \\
\text { HR: } 125 \mathrm{bpm} \\
\text { SBP: } 70 \mathrm{~mm} \mathrm{Hg} \\
\text { DBP: } 41 \mathrm{~mm} \mathrm{Hg} \\
\text { Spo }_{2}: 70\end{array}$ & $\begin{array}{l}1 \mathrm{~mm} \text { ST elevation } \\
\text { in leads V2-V6 with } \\
\text { associated Q waves in } \\
\text { leads V4-V6 }\end{array}$ & $\begin{array}{l}\text { WBC: - } \\
\text { Lymphocyte: - } \\
\text { CRP: - } \\
\text { D-dimer: - } \\
\text { Troponin: } 1.6 \mathrm{ng} / \mathrm{ml} \\
\text { BNP: - }\end{array}$ \\
\hline $\begin{array}{l}\text { Case 13, } \\
\text { Juusela } \\
\text { et al. }{ }^{15}\end{array}$ & $\begin{array}{l}45, \mathrm{~F} \\
\text { Preg- } \\
\text { nant ( } 39 \\
\text { weeks) }\end{array}$ & $\begin{array}{l}\text { Obesity, } \\
\text { gestational } \\
\text { diabetic }\end{array}$ & $\begin{array}{l}\text { Contractions and } \\
\text { emesis }\end{array}$ & $\begin{array}{l}\text { Fever: } 99.6 \text { F } \\
\text { HR: } 120 \mathrm{bpm} \\
\text { SBP: } 183 \mathrm{~mm} \mathrm{Hg} \\
\text { DBP: } 114 \mathrm{~mm} \mathrm{Hg} \\
\mathrm{SpO}_{2}: 96\end{array}$ & $\begin{array}{l}\text { Nonspecific T-wave } \\
\text { abnormalities }\end{array}$ & $\begin{array}{l}\text { WBC: - } \\
\text { Lymphocyte: - } \\
\text { CRP: - } \\
\text { D-dimer: - } \\
\text { Troponin: } 0.046 \text { ng/mL } \\
\text { BNP: } 114 \text { pg/mL }\end{array}$ \\
\hline $\begin{array}{l}\text { Case 14, } \\
\text { Juusela } \\
\text { et al. }^{15}\end{array}$ & $\begin{array}{l}26, \mathrm{~F} \\
\text { Preg- } \\
\text { nant (33 } \\
\text { weeks) }\end{array}$ & $\begin{array}{l}\text { Obesity, } \\
\text { polycystic } \\
\text { ovary } \\
\text { syndrome }\end{array}$ & $\begin{array}{l}\text { Shortness of breath, } \\
\text { dyspnea, }\end{array}$ & $\begin{array}{l}\text { Fever: } 99.6 \text { F } \\
\text { HR: } 130 \mathrm{bpm} \\
\text { SBP: } 110 \mathrm{~mm} \mathrm{Hg} \\
\text { DBP: } 70 \mathrm{~mm} \mathrm{Hg} \\
\text { Spo }_{2}: 95\end{array}$ & $\begin{array}{l}\text { Supraventricular } \\
\text { tachycardia }\end{array}$ & $\begin{array}{l}\text { WBC: - } \\
\text { Lymphocyte: - } \\
\text { CRP: } 7.68 \mathrm{mg} / \mathrm{dL} \\
\text { D-dimer: - } \\
\text { Troponin: } 0.046 \mathrm{ng} / \mathrm{mL} \text {, } \\
\text { BNP: }<10 \mathrm{pg} / \mathrm{mL} \text {, }\end{array}$ \\
\hline $\begin{array}{l}\text { Case } 15, \\
\text { Pavon } \\
\text { et al. }^{16}\end{array}$ & $64, M$ & $\begin{array}{l}\text { Pulmonary } \\
\text { sarcoidosis } \\
\text { and epilepsy }\end{array}$ & $\begin{array}{l}\text { Chest pain and } \\
\text { dyspnea. }\end{array}$ & $\begin{array}{l}\text { Fever: } 39.3^{\circ} \mathrm{C} \\
\text { HR: - } \\
\text { SBP: - } \\
\text { DBP: - } \\
\text { SpO2: - }\end{array}$ & Unremarkable & $\begin{array}{l}\text { WBC: } 18.7 \mathrm{gr} / \mathrm{L} \\
\text { Lymphocyte: - } \\
\text { CRP: - } \\
\text { D-dimer: } 1210 \mathrm{ng} / \mathrm{ml} \\
\text { Troponin: } 1843 \mathrm{ng} / \mathrm{L} \\
\text { BNP: - }\end{array}$ \\
\hline $\begin{array}{l}\text { Case 16, } \\
\text { Irabien- } \\
\text { Ortiz et al. }{ }^{17}\end{array}$ & $59, F$ & $\begin{array}{l}\text { Hypertension, } \\
\text { cervical } \\
\text { degenerative } \\
\text { arthropathy, } \\
\text { chronic lum- } \\
\text { bar radiculop- } \\
\text { athy, } \\
\text { erythema } \\
\text { nodosum, } \\
\text { migraine }\end{array}$ & $\begin{array}{l}\text { Anginal chest pain } \\
\text { in the absence of respi- } \\
\text { ratory symptoms. }\end{array}$ & $\begin{array}{l}\text { Fever: } 39.3^{\circ} \mathrm{C} \\
\text { HR: - } \\
\text { SBP: } 75 \mathrm{~mm} \mathrm{Hg} \\
\text { DBP: } 53 \mathrm{~mm} \mathrm{Hg} \\
\text { SpO }_{2}: 96\end{array}$ & $\begin{array}{l}\text { Concave ST-segment } \\
\text { elevation and PR-seg- } \\
\text { ment depression, as } \\
\text { well } \\
\text { as low voltages }\end{array}$ & $\begin{array}{l}\text { WBC: } 14.17 \times 10^{9} / \mathrm{L} \\
\text { Lymphocyte: } 2.59 \times 10^{9} / \mathrm{L} \\
\text { CRP: - } \\
\text { D-dimer: } 23.242 \mathrm{ng} / \mathrm{mL} \\
\text { Troponin: Elevated } \\
\text { BNP: } 4421 \mathrm{ng} / \mathrm{L}\end{array}$ \\
\hline
\end{tabular}

Abbreviations: F; female, M; male, HR; heart rate, SBP; systolic blood pressure, DBP; diastolic blood pressure, WBC; white blood cell, CRP; c-reactive protein, BNP; brain natriuretic peptide.

TABLE 2. IMAGING FINDINGS, IN-HOSPITAL TREATMENT, AND COURSE OF ALL COVID-19 ACUTE MYOCARDITIS PATIENTS

\begin{tabular}{|c|c|c|c|c|c|}
\hline $\begin{array}{l}\text { Case, } \\
\text { author }\end{array}$ & $\begin{array}{l}\text { Echocardiographic } \\
\text { findings }\end{array}$ & CMR imaging findings & $\begin{array}{l}\text { EMB } \\
\text { findings }\end{array}$ & In-hospital treatment & $\begin{array}{l}\text { In-hospital } \\
\text { course }\end{array}$ \\
\hline $\begin{array}{l}\text { Case 1, } \\
\text { Inciardi } \\
\text { et al. }{ }^{4}\end{array}$ & $\begin{array}{l}\text { Diffuse hypokinesia, } \\
\text { with an estimated left } \\
\text { ventricle ejection fraction } \\
\text { of } 40 \%\end{array}$ & $\begin{array}{l}\text { Diffuse biventricular hypokinesia, } \\
\text { especially in the apical segments, and } \\
\text { severe LV dysfunction }\end{array}$ & - & $\begin{array}{l}\text { Hydroxychloroquine (200 mg twice } \\
\text { daily), lopinavir/ritonavir ( } 2 \text { tablets } \\
\text { of } 200 / 50 \mathrm{mg} \text { twice daily), and } \\
\text { intravenous methylprednisolone (1 } \\
\mathrm{mg} / \mathrm{kg} \text { daily for), } 50 \mathrm{mg} \text { of kanre- } \\
\text { none, } 25 \text { to } 50 \mathrm{mg} \text { of furosemide, } \\
\text { and } 2.5 \mathrm{mg} \text { of bisoprolol }\end{array}$ & $\begin{array}{l}\text { Cardiogenic } \\
\text { shock, } \\
\text { clinical } \\
\text { follow-up }\end{array}$ \\
\hline $\begin{array}{l}\text { Case 2, } \\
\text { Kim } \\
\text { et al. }{ }^{5}\end{array}$ & $\begin{array}{l}\text { Severe left ventricular } \\
\text { systolic dysfunction }\end{array}$ & $\begin{array}{l}\text { Diffuse high signal intensity in the } \\
\text { left ventricle myocardium on } \mathrm{T} 2 \text { short } \\
\text { inversion recovery image }\end{array}$ & - & - & Discharged \\
\hline $\begin{array}{l}\text { Case } 3 \text {, } \\
\text { Zeng } \\
\text { et al. }{ }^{6}\end{array}$ & $\begin{array}{l}\text { An enlarged left ventricle } \\
(61 \mathrm{~mm}) \text {, diffuse myo- } \\
\text { cardial dyskinesia along } \\
\text { with a low left ventricular } \\
\text { ejection fraction of } 32 \%\end{array}$ & - & - & $\begin{array}{l}\text { High-flow oxygen, lopinavir-ritona- } \\
\text { vir, interferon } \alpha-1 b \text {, methylprednis- } \\
\text { olone, immunoglobulin, piperacil- } \\
\text { lin-tazobactam, and continuous } \\
\text { renal replacement therapy }\end{array}$ & $\begin{array}{l}\text { Cardiogenic } \\
\text { shock, } \\
\text { exitus }\end{array}$ \\
\hline $\begin{array}{l}\text { Case } 4, \\
\text { Doyen } \\
\text { et al. }{ }^{7}\end{array}$ & $\begin{array}{l}\text { Mild left ventricle hyper- } \\
\text { trophy, the left ventricu- } \\
\text { lar ejection fraction and } \\
\text { wall motion were within } \\
\text { normal limits }\end{array}$ & $\begin{array}{l}\text { Subepicardial late gadolinium en- } \\
\text { hancement of the apex and inferolater- } \\
\text { al wall-suggestive of myocarditis }\end{array}$ & - & $\begin{array}{l}\text { Hydrocortisone, aspirin, } \\
\text { fondaparinux }\end{array}$ & $\begin{array}{l}\text { Acute } \\
\text { respiratory } \\
\text { distress } \\
\text { syndrome, } \\
\text { discharged }\end{array}$ \\
\hline
\end{tabular}


TABLE 2.

(CONCLUSION)

\begin{tabular}{|c|c|c|c|c|c|}
\hline $\begin{array}{l}\text { Case, } \\
\text { author }\end{array}$ & $\begin{array}{l}\text { Echocardiographic } \\
\text { findings }\end{array}$ & CMR imaging findings & $\begin{array}{l}\text { EMB } \\
\text { findings }\end{array}$ & In-hospital treatment & $\begin{array}{l}\text { In-hospital } \\
\text { course }\end{array}$ \\
\hline $\begin{array}{l}\text { Case 5, } \\
\text { Trogen } \\
\text { et al. }{ }^{8}\end{array}$ & $\begin{array}{l}\text { Left ventricular ejection } \\
\text { fraction qualitatively not- } \\
\text { ed to be mildly depressed } \\
\text { without obvious intrac- } \\
\text { ardiac clots or pericardial } \\
\text { effusion }\end{array}$ & $\begin{array}{l}\text { Normal size left ventricle (LV) with } \\
\text { mildly decreased systolic function } \\
\text { (40\%) and normal right ventricular } \\
\text { (RV) size with mildly diminished } \\
\text { systolic function (RVEF of } 39 \%) \text {. There } \\
\text { was an area of mid-wall late gado- } \\
\text { linium enhancement at the inferior } \\
\text { LV-RV junction corresponding to an } \\
\text { area of increased T2 signal, as well as } \\
\text { an area of hypokinesia }\end{array}$ & - & $\begin{array}{l}\text { Hydroxychloroquine, piperacillin/ } \\
\text { tazobactam, enoxaparin }\end{array}$ & Discharged \\
\hline $\begin{array}{l}\text { Case 6, } \\
\text { Sardari } \\
\text { et al. }{ }^{9}\end{array}$ & $\begin{array}{l}\text { Mild left ventricular } \\
\text { dysfunction }\end{array}$ & $\begin{array}{l}\text { Normal left ventricular size with a } \\
\text { mildly reduced ejection fraction of } \\
50 \% . \text { T2-weighted sequence with its } \\
\text { post-analysis showed edema/inflam- } \\
\text { mation in the mid inferoseptal and } \\
\text { inferior wall. Late gadolinium enhance- } \\
\text { ment showed subepicardial fibrosis in } \\
\text { the mid inferior wall }\end{array}$ & - & Bisoprolol and lisinopril & Discharged \\
\hline $\begin{array}{l}\text { Case } 7, \\
\text { Coyle } \\
\text { et al. }{ }^{10}\end{array}$ & $\begin{array}{l}\text { Moderate diffuse hypoki- } \\
\text { nesia with relative apical } \\
\text { sparing and a left ventric- } \\
\text { ular ejection fraction of } \\
35-40 \%\end{array}$ & $\begin{array}{l}\text { Diffuse bi-ventricular and bi-atrial } \\
\text { edema with a small area of late gado- } \\
\text { linium enhancement }\end{array}$ & - & $\begin{array}{l}\text { Hydroxychloroquine, azithromycin, } \\
\text { ceftriaxone, methylprednisolone, } \\
\text { colchicine }\end{array}$ & Discharged \\
\hline $\begin{array}{l}\text { Case 8, } \\
\text { Beşler } \\
\text { and } \\
\text { Arslan }{ }^{11}\end{array}$ & - & $\begin{array}{l}\text { Short tau inversion recovery (STIR) } \\
\text { sequence revealed a subepicardial high } \\
\text { signal intensity in the mid postero- } \\
\text { lateral wall of the left ventricle which } \\
\text { suggests myocardial wall edema }\end{array}$ & - & $\begin{array}{l}\text { Hydroxychloroquine, azithromycin, } \\
\text { ceftriaxone, tigecycline, favipiravir, } \\
\text { colchicine }\end{array}$ & Discharged \\
\hline $\begin{array}{l}\text { Case 9, } \\
\text { Yuan } \\
\text { et al. }{ }^{12}\end{array}$ & - & $\begin{array}{l}\text { The signal of T2 weighted image in the } \\
\text { apical region of the left ventricle was } \\
\text { increased, which indicated the possi- } \\
\text { bility of myocardial cell edema. } \\
\text { Left ventricular systolic function was } \\
\text { slightly decreased }\end{array}$ & - & - & - \\
\hline $\begin{array}{l}\text { Case } 10, \\
\text { Cizgici } \\
\text { et al. }\end{array}$ & $\begin{array}{l}\text { Not being able to per- } \\
\text { form echocardiography }\end{array}$ & $\begin{array}{l}\text { Not being able to perform magnetic } \\
\text { resonance imaging }\end{array}$ & - & $\begin{array}{l}\text { Furosemide, beta-blocker, and } \\
\text { angiotensin-converting enzyme } \\
\text { inhibitor was added to his Covid-19 } \\
\text { specific therapy. }\end{array}$ & - \\
\hline $\begin{array}{l}\text { Case 11, } \\
\text { Asif and } \\
\text { Ali14 }\end{array}$ & $\begin{array}{l}\text { Normal left ventricle } \\
\text { ejection fraction of } 70- \\
75 \% \text { with no regional wall } \\
\text { motion abnormalities }\end{array}$ & - & - & $\begin{array}{l}\text { Aspirin } 81 \text { mg, clopidogrel } 75 \text { mg, } \\
\text { heparin, azithromycin, hydroxychlo- } \\
\text { roquine, meropenem, tocilizumab, } \\
\text { norepinephrine, phenylephrine, } \\
\text { vasopressin, atracurium, propofol, } \\
\text { fentanyl }\end{array}$ & Intubated \\
\hline $\begin{array}{l}\text { Case 12, } \\
\text { Asif and } \\
\text { Ali }{ }^{14}\end{array}$ & $\begin{array}{l}\text { Normal left ventricle } \\
\text { ejection fraction of } \\
65-70 \% \text { with no regional } \\
\text { wall motion abnormal- } \\
\text { ities }\end{array}$ & - & & $\begin{array}{l}\text { Aspirin } 81 \text { mg, clopidogrel } 75 \text { mg, } \\
\text { heparin, azithromycin, cefepime, } \\
\text { vancomycin, tocilizumab, norepi- } \\
\text { nephrine, phenylephrine, midazol- } \\
\text { am, and fentanyl }\end{array}$ & Intubated \\
\hline $\begin{array}{l}\text { Case } 13, \\
\text { Juusela } \\
\text { et al. }{ }^{15}\end{array}$ & $\begin{array}{l}\text { Moderately reduced left } \\
\text { ventricular ejection frac- } \\
\text { tion of } 40 \% \text { with global } \\
\text { hypokinesia }\end{array}$ & - & - & $\begin{array}{l}\text { IV methylprednisolone, hydroxy- } \\
\text { chloroquine, tocilizumab } \\
\text {. }\end{array}$ & $\begin{array}{l}\text { Intubated, } \\
\text { (a primary } \\
\text { cesarean) }\end{array}$ \\
\hline $\begin{array}{l}\text { Case 14, } \\
\text { Juusela } \\
\text { et al. }{ }^{15}\end{array}$ & $\begin{array}{l}\text { Moderately reduced } \\
\text { left ventricular ejection } \\
\text { fraction of } 40-45 \% \text { with } \\
\text { global hypokinesia }\end{array}$ & - & - & Metoprolol & Cesarean \\
\hline $\begin{array}{l}\text { Case } 15, \\
\text { Pavon } \\
\text { et al. }{ }^{16}\end{array}$ & $\begin{array}{l}\text { Moderately reduced } \\
\text { left ventricular ejection } \\
\text { fraction of } 47 \% \text {. }\end{array}$ & $\begin{array}{l}\text { T2-mapping sequences showed } \\
\text { myocardial edema and sub-epicardial } \\
\text { late gadolinium enhancement in the } \\
\text { anterior interventricular septum, in the } \\
\text { inferior and inferolateral walls }\end{array}$ & - & Piperacillin/tazobactam & Discharged \\
\hline $\begin{array}{l}\text { Case 16, } \\
\text { Irabien- } \\
\text { Ortiz } \\
\text { et al. }{ }^{17}\end{array}$ & $\begin{array}{l}\text { Preserved left ventricular } \\
\text { ejection fraction without } \\
\text { segmental abnormalities, } \\
\text { and moderate pericardial } \\
\text { effusion with no clear } \\
\text { signs of hemodynamic } \\
\text { deterioration }\end{array}$ & - & - & $\begin{array}{l}\text { Immunoglobulins }(80 \mathrm{mg} / \mathrm{d}) \text { meth- } \\
\text { ylprednisolone }(500 \mathrm{mg} / \mathrm{d}) \text {, antiviral } \\
\text { treatment consisting of interferon-B } \\
(0.25 \mathrm{mg} / 48 \mathrm{~h}) \text { and ritonavir/lopina- } \\
\operatorname{vir}(400 \mathrm{mg} / 100 \mathrm{mg} / 12 \mathrm{~h})\end{array}$ & $\begin{array}{l}\text { Cardiogenic } \\
\text { shock }\end{array}$ \\
\hline
\end{tabular}

Abbreviations: CMR; cardiac magnetic resonance, EMB; endomyocardial biopsy 
amelioration using IV glucocorticoid and immunoglobulin therapy. This finding appears as a clue highlighting the cytokine storm due to inadequate host immune response, which might be the main pathophysiology of COVID-19-induced acute fulminant myocarditis.

\section{Limitations in the diagnosis of COVID-19-re-}

\section{lated acute myocarditis}

Acute viral infections are one of the most common etiologic factors of acute myocarditis. Following the COVID-19 pandemic, several cases of acute myocarditis were reported worldwide, which were diagnosed with different modalities other than EMB. In our review, all of the published cases were mentioned in terms of clinical characteristics, imaging findings, and in-hospital course.

Troponin has been indicated as an independent predictor of mortality in hospitalized patients with COVID-19 ${ }^{18,19}$. Since troponin has been defined as a noteworthy prognostic factor, the etiology of the higher levels of troponin gains clinical importance to regulate adequate medications. In COVID-19 patients who present with elevated troponin levels, it might be difficult to reach a definitive diagnosis of acute coronary syndrome (ACS) or acute myocarditis because there are similarities among them in regard to the elevation of troponin levels and ECG changes. It is reasonable not to perform CMR and MRI in most patients due to the contiguousness of COVID-19. On the other hand, underusing the aforementioned modalities in the differential diagnosis reveals a gap in the definite etiology of the myocardial injury. Thus, we may be underestimating the prevalence and importance of
COVID-19-related acute myocarditis. Moreover, in severe patients, both ACS and acute myocarditis may present together because of the procoagulant and inflammatory nature of the COVID-19 infection.

\section{CONCLUSION}

Despite the COVID-19 pandemic worldwide, a limited number of cases has been shared in the current literature. There are a lot of difficulties for the differential diagnosis of acute myocarditis in the context of COVID-19, and information about COVID-19-related acute myocarditis remains unclear. Also, there is no consensus about the diagnostic and treatment algorithms in patients with COVID-19-induced acute myocarditis. Hence, further studies and case reports on COVID-19-associated acute myocarditis are needed to clarify an appropriate approach to these patients.

\section{Conflict of interest}

The authors have no conflicts of interest relevant for this article

\section{Funding}

The authors declare that this article has received no financial support.

\section{Author's Contribution}

Concept: T.Ç., M.I.H.; Design: T.Ç., M.İ.H.; Supervision: M.U., A.L.O.; Funding: V.Ç.; Materials: T.Ç., M.İ.H., V.Ç.; Data collection: T.Ç., V.Ç.; Analysis: M.İ.H.; Literature review: T.Ç., M.İ.H., V.Ç.; Writing: T.Ç.; Critical review: M.U., A.L.O.

\section{RESUMO}

INTRODUÇão: Na literatura atual, houve um aumento dos casos apresentados com doença coronavírus de 2019 (COVID-19) induzida por miocardite aguda. Nesta revisão baseada em casos, buscamos descrever as características clínicas, achados de imagem e curso hospitalar de miocardite aguda. Além disso, as limitações em relação ao diagnóstico de miocardite foram discutidas, uma vez que apenas casos de miocardite fulminante foram mencionados na literatura atual.

MÉTODOs: Fizemos uma revisão da literatura de todos os pacientes diagnosticados com miocardite aguda induzida por COVID-19 com a utilização das bases de dados PubMed, Embase e Cochrane.

RESULTADO: Dezesseis casos relatados estão relacionados com a miocardite aguda induzida pela COVID-19. Observamos que os achados de ECG na maioria dos pacientes com COVID-19 não eram específicos, incluindo elevação difusa do segmento ST, atraso não específico da condução intraventricular, taquicardia sinusal e ondas T invertidas em pistas anteriores. Os resultados ecocardiográficos de doentes com miocardite aguda COVID-19 variaram entre a fração de ejeção ventricular esquerda preservada (LVEF) sem anomalias segmentais e a LVEF reduzida com hipocinésia global. Curiosamente, alguns pacientes com COVID-19 induzidos à miocardite aguda fulminante eram sensiveis aos esteroides e tinham uma melhoria com glucocorticoides e terapia com imunoglobulina.

CONCLUSÃO: Apesar da pandemia de COVID-19 em todo o mundo, um número limitado de casos tem sido compartilhado na literatura atual. Há muitas dificuldades para o diagnóstico diferencial de miocardite aguda no contexto da COVID-19. 


\section{REFERENCES}

1. Zhou F, Yu T, Du R, Fan G, Liu Y, Liu Z, et al. Clinical course and risk factors for mortality of adult inpatients with COVID-19 in Wuhan, China: a retrospective cohort study. Lancet. 2020;395(10229):1054-62.

2. Bavishi C, Bonow RO, Trivedi V, Abbott JD, Messerli FH, Bhatt DL. Acute myocardial injury in patients hospitalized with COVID-19 infection: a review. Prog Cardiovasc Dis. 2020;S0033-0620(20)30123-7.

3. Lang JP, Wang X, Moura FA, Siddiqi HK, Morrow DA, Bohula EA. A current review of COVID-19 for the cardiovascular specialist. Am Heart !. 2020;226:29-44

4. Inciardi RM, Lupi L, Zaccone G, Italia L, Raffo M, Tomasoni D, et al. Cardiac Involvement in a patient with coronavirus disease 2019 (COVID-19). JAMA Cardiol. 2020;5(7):1-6.

5. Kim IC, Kim JY, Kim HA, Han S. COVID-19-related myocarditis in a 21-yearold female patient. Eur Heart J. 2020;41(19):1859.

6. Zeng JH, Liu YX, Yuan J, Wang FX, Wu WB, Li IX, et al. First case of COVID19 complicated with fulminant myocarditis: a case report and insights. Infection. 2020;1-5.

7. Doyen D, Moceri P, Ducreux D, Dellamonica J. Myocarditis in a patient with COVID-19: a cause of raised troponin and ECG changes. Lancet. 2020;395(10235):1516.

8. Trogen B, Gonzalez FJ, Shust GF. COVID-19-associated myocarditis in an adolescent. Pediatr Infect Dis J. 2020;39(8):e204-5.

9. Sardari A, Tabarsi P, Borhany H, Mohiaddin R, Houshmand G. Myocarditis detected after COVID-19 recovery. Eur Heart J Cardiovasc Imaging. 2020;jeaa166.

10. Coyle J, Igbinomwanhia E, Sanchez-Nadales A, Danciu S, Chu C, Shah $\mathrm{N}$. A recovered case of COVID-19 myocarditis and ARDS treated with corticosteroids, tocilizumab, and experimental AT-001. JACC Case Rep. 2020;2(9):1331-6.
11. Beşler MS, Arslan H. Acute myocarditis associated with COVID-19 infection. Am I Emerg Med. 2020;S0735-6757(20)30463-0.

12. Yuan WF, Tang X, Zhao XX. An 'asymptomatic' driver with COVID-19: atypical suspected myocarditis by SARS-CoV-2. Cardiovasc Diagn Ther. 2020;10(2):242-3.

13. Cizgici AY, Zencirkiran Agus H, Yildiz M. COVID-19 myopericarditis: it should be kept in mind in today's conditions. Am J Emerg Med. 2020;38(7):1547. e5-1547.e6.

14. Asif T, Ali Z. Transient ST segment elevation in two patients with COVID-19 and a normal transthoracic echocardiogram. Eur J Case Rep Intern Med. 2020;7(5):001672

15. Juusela A, Nazir M, Gimovsky M. Two cases of coronavirus 2019-related cardiomyopathy in pregnancy. Am J Obstet Gynecol MFM. 2020;2(2):100113.

16. Pavon AG, Meier D, Samim D, Rotzinger DC, Fournier S, Marquis P, et al. First documentation of persistent SARS-CoV-2 Infection presenting with late acute severe myocarditis. Can | Cardiol. 2020;S0828-282X(20)30532-8.

17. Irabien-Ortiz A, Carreras-Mora J, Sionis A, Pàmies J, Montiel J, Tauron M. Fulminant myocarditis due to COVID-19. Rev Esp Cardiol (Engl Ed). 2020;73(6):503-4.

18. Nie SF, Yu M, Xie T, Yang F, Wang HB, Wang ZH, et al. Cardiac troponin $I$ is an independent predictor for mortality in hospitalized patients with coronavirus disease 2019. Circulation. 2020. doi: 10.1161/ CIRCULATIONAHA.120.048789.

19. Imazio $M$, Klingel $K$, Kindermann I, Brucato A, De Rosa FG, Adler $Y$, et al. COVID-19 pandemic and troponin: indirect myocardial injury, myocardial inflammation or myocarditis? Heart. 2020; heartjnl-2020-317186. doi: 10.1136/heartjnl-2020-317186. 